\title{
Studying the phenomena related to the IR thermographic detection of buried landmines
}

\author{
by V.P. Vavilov and A. G. Klimov
}

Tomsk Polytechnic University, Institute of Introscopy, Russia, 634028, Tomsk, 28, Savinykh St., 3, E-mail: vavilov@introscopy.tpu.ru

\begin{abstract}
Some theoretical and experimental problems related to the IR detection of buried landmines are discussed to express a rather pessimistic conclusion on the statistical reliability of this technique.
\end{abstract}

\section{Introduction}

Infrared (IR) thermographic detection of buried landmines has been considered as a promising diagnostic tool for many years. The number of related papers is large, including the information about the successful practical use of this technique by the U.S. Army in Kuwait during the "Desert Storm" operation. However, our fragmentary experience in this application field shows that the problem is not yet exhausted, in particular, if to discuss its statistical reliability. It is known that thermal NDT of materials is accompanied by a high level of specific noise that transits in either a high false alarm or a low correct detection. When detecting landmines, this statement is more than true, because, in this case, the number of factors influencing surface temperature gradients is enormous.

In this study, we have analyzed some theoretical and practical issues related to the IR detection of buried landmines. A couple of models have been used to evaluate the influence of some non-trivial factors, such as wind, cloudiness, use of an artificial heater, etc.

The experiments have been performed on two mine surrogates made of aluminum and paraffin.

\section{Advanced numerical model of buried landmines}

The term "advanced model" used in this paper is confronted to the term "classical model" that follows from many studies where a buried mine is treated as a "defect" or foreign object in a soil matrix.

When modeling the detection of buried landmines, both heat and mass transfer phenomena must be analyzed. The soil moisture content, and, hence, the soil thermal properties, all vary with time, and not always in a straightforward manner. Surface evaporation depends on relative humidity, temperature and wind speed. Evaporation may change surface temperature gradients. The solar radiation that is the thermal stimulus in the detection of buried mines is not a simple cosine function as it is usually expressed in simple modeling. Cloudy periods, the change in the angle of solar radiation, shadows caused by nearby objects, or surface irregularities require modeling solar radiation input as a complex function. Also, surface heat exchange is strongly dependent on wind and both soil and air temperature. Furthermore, the great variety of mine types and designs, such as a mine in a protective aluminum case, leads to unusual "defect" geometries.

Antipersonnel and antitank landmines are typically modeled as homogeneous disks located in uniform soil at a depth from 1 to $15 \mathrm{~cm}$. Thus, the related geometry is cylindrical (two-dimensional - 2D). The soil is heated by solar radiation, in a diurnal cycle simulated by: $Q(\tau)=Q_{m}[1+\cos (\omega \tau)]$, where the cyclic frequency $\omega$ is related to $24 \mathrm{hr}$ variations. Most researchers believe that the detection of buried mines is possible primarily due to changes in the thermal properties of the soil appearing when installing a landmine. 
A classical 2D cylindrical model allows the determination of the $\Delta T=T_{\mathrm{d}}-T_{\text {nd }}$ signal as a function of time, mine depth and size, thermal properties. Here: $T_{\mathrm{d}}$ is the temperature over the center of a mine, and $T_{\text {nd }}$ is the temperature over an area where there is no mine. Because of the low thermal conductivity of explosives, the soil over a case-free mine is warmer than the surrounding soil during the daytime $(\Delta T>0)$ reaching few degrees centigrade in the middle of the day. Shallow mines may cause an inversion in $\Delta T(\Delta T<0)$ during the night. Such classical model was analyzed in [1] to show that the mine depth $l$ is the most important factor influencing both the values of signal magnitude $\Delta T$ and the optimal observation time $\tau_{\mathrm{m}}$. The example of the "classical" depth calibration curve is shown in figure 1 for the trinitrotoluene (TNT) explosive buried in soil. In Section 3 this curve is used to determine the depth detection limit by comparing the $\Delta T$ values and the noise level.

The advanced numerical model of which details are described in [1] allows analyzing the influence of: 1) a heating profile, 2) soil moisture, and 3) a mine case. Another advanced model proposed in [2] enables the study of surface roughness and water mass transfer. Here we shall limit ourselves with some illustrations following from the results described in [1].

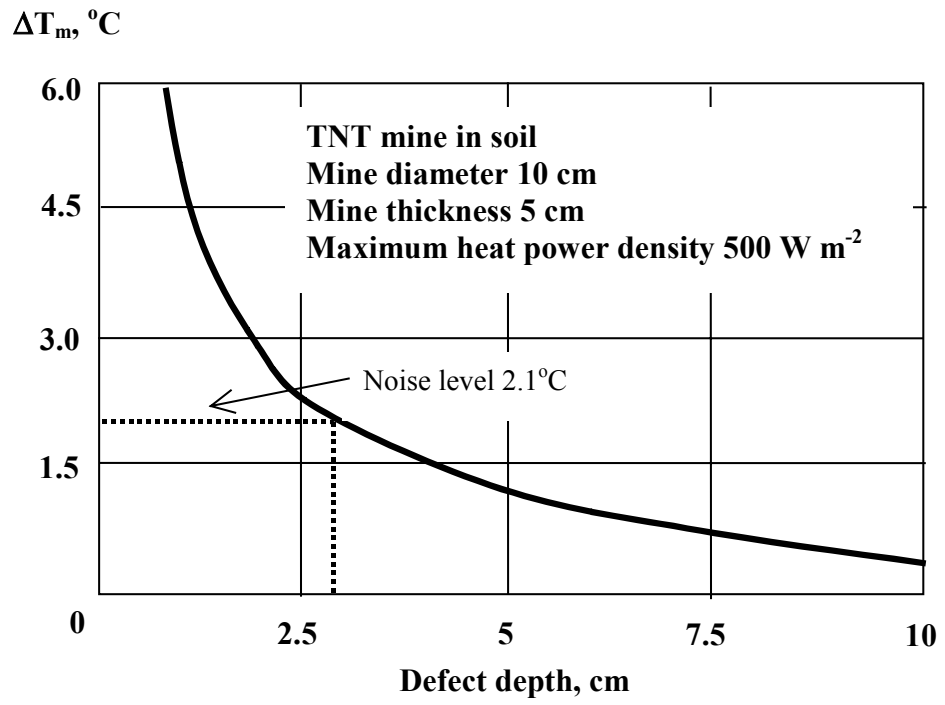

$\begin{aligned} \text { Fig. 1. Depth calibration function - trinitrotoluene: } & \lambda=0.23 \mathrm{~W} \mathrm{~m}^{-1} \mathrm{~K}^{-1} ; a=0.0910^{-6} \mathrm{~m}^{2} \mathrm{~s}^{-1} \\ \text { - soil: } & \lambda=2.6 \mathrm{~W} \mathrm{~m}^{-1} \mathrm{~K}^{-1} ; a=0.510^{-6} \mathrm{~m}^{2} \mathrm{~s}^{-1}\end{aligned}$

\subsection{Heating profile}

There are several heating profiles presented in figure 2 along with the corresponding $\Delta T_{\mathrm{m}}$ and $\tau_{\mathrm{m}}$ values calculated by using the $2 \mathrm{D}$ advanced model. The basic profile of the heat power density is given with the solid line (figure 2a), and some disturbing factors are shown with the dashed lines (figure $2 b-e$ ). The calculations are performed by using the ThermoHeat-3DPro software from Tomsk Polytechnic University [1]. The following phenomena are illustrated by figure $2 b-e: 1)$ the sudden sharp increase of the wind velocity that results in the increase of the heat exchange coefficient (figure $2 b$ ), 2) the increase of the ambient temperature during daytime (figure $2 c$ ), 3 ) the sudden drop of solar radiation (cloudy sky, figure $2 d$ ), and 4 ) the additional stimulation of soil with an 
artificial source (figure 2e). It is seen that the surface temperatures are mainly influenced by the wind and the use of the artificial heat source.

\subsection{Soil moisture}

Soil moisture influences on the detectability of mines in a contradictory way. First, the rainwater is typically colder than the soil, so a rain cools the soil and obliterates thermal patterns. Second, moisture changes the thermal properties of a soil. Third, surface evaporation adds a negative heat flux to the soil surface. Fourth, moisture changes the soil emissivity. Some examples of how soil moisture influences $\Delta T$ are given in [1].

\subsection{A landmine in a case}

Modeling has been made by using the ThermoCalc-2DM program [1]. A $10 \mathrm{~cm}$ diameter case-free TNT landmine that is in perfect contact with the soil produces the $\Delta T_{\mathrm{m}}=2.49^{\circ} \mathrm{C}$ at $1 \mathrm{PM}$. The same mine in a $3 \mathrm{~mm}$-thick aluminum case produces the much lower signal, $\Delta T_{\mathrm{m}}=1.42^{\circ} \mathrm{C}$ that is explained by a high conductivity of aluminum. If the TNT mine were surrounded by air rather than by aluminum, the signal would increase to $\Delta T_{\mathrm{m}}=2.84^{\circ} \mathrm{C}$.

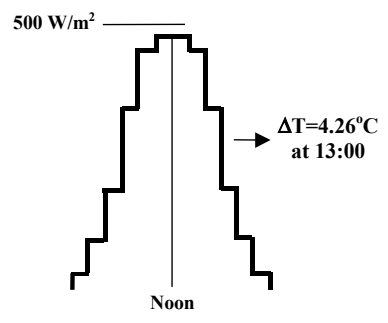

a)

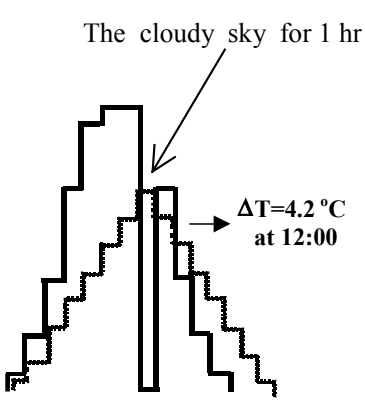

d)

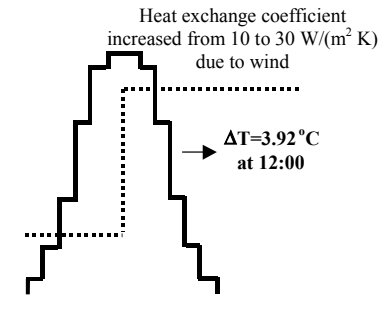

b)

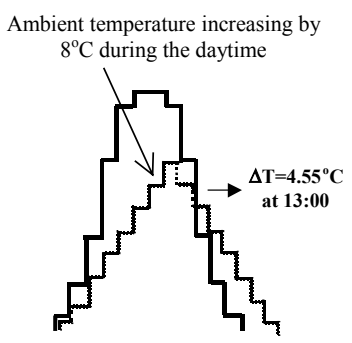

c)

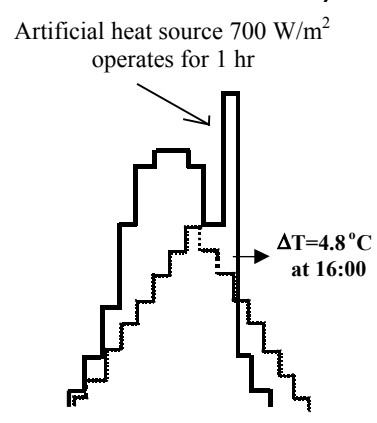

e)

Fig. 2. Influence of a heating profile on the maximum temperature signal $\Delta \mathrm{T}_{m}$ and the optimal observation time $\tau_{m}$ (TNT in soil, mine diameter $20 \mathrm{~cm}$, mine thickness $10 \mathrm{~cm}$, mine depth $5 \mathrm{~cm}$ )

\section{Experimental results}

In Siberia, the summertime solar radiation ensures only from 300 to $500 \mathrm{~W} \mathrm{~m}^{-2}$ of absorbed energy, therefore the landmine surrogates (see figure 3) have been placed in both sand and soil at depths not more than few centimeters. The absorbed power density 
$Q_{\text {abs }}$ of the solar radiation was measured by placing a flux meter within a soil surface layer. Most experimental data have been related to the maximum solar radiation flux $Q_{m} \approx 350$ $\mathrm{Wm}^{-2}$.
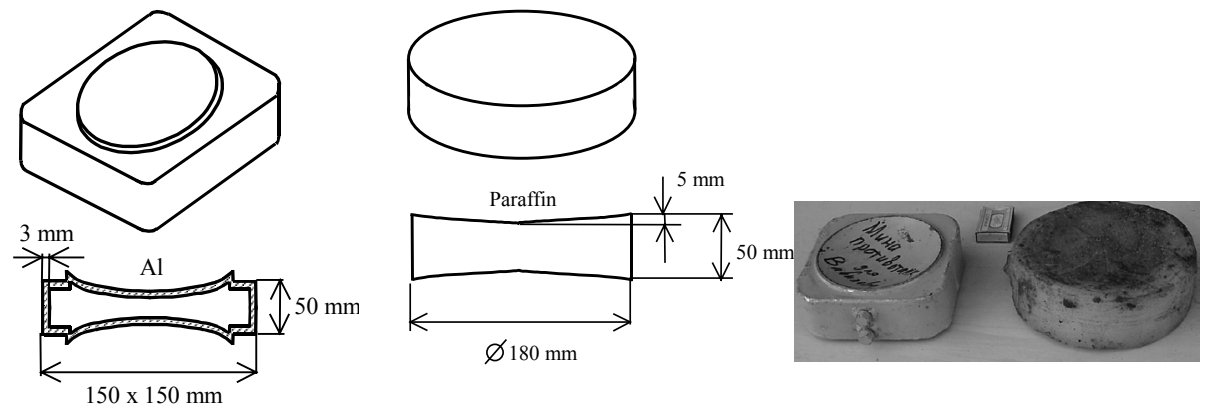

Fig. 3. Landmine surrogates

\subsection{Surface temperature noise and depth detection limit}

A natural variation of the temperature on the soil surface is characterized by the noise standard variation $\sigma_{\mathrm{n}},{ }^{\circ} \mathrm{C}$. The corresponding values have been measured for several types of soil (see table 1) by using a Thermovision 570 IR camera. As expected, the lowest noise adheres to uniform materials, such as asphalt, concrete, etc. Uneven surface, e.g. alternating soil and vegetation, are characterized by a high $\sigma_{\mathrm{n}}$ value.

Let us assume that a landmine can be reliably detected if a surface temperature signal exceeds $2 \sigma_{\mathrm{n}}$. Compare the data in table 1 and figure 1 for the soil with a short poor vegetation $\left(\sigma_{n}=0.75^{\circ} \mathrm{C}\right)$. In the case of $Q_{m}=500 \mathrm{~W} \mathrm{~m}^{-2}$, the threshold noise level will be $2 \cdot 0.75^{\circ} \mathrm{C} \cdot(500 / 350)=2.1^{\circ} \mathrm{C}$ (temperature signals are linearly proportional to absorbed energy). Therefore, according to figure 1 , a TNT landmine (diameter $10 \mathrm{~cm}$, thickness 5 $\mathrm{cm}$ ) will be reliably detected up to depths of about $3 \mathrm{~cm}$.

Table 1: Surface temperature noise (maximal solar radiation $Q_{m} \approx 350 \mathrm{Wm}^{-2}$ )

\begin{tabular}{|l|c|}
\hline \multicolumn{1}{|c|}{ Type of soil } & $\sigma_{\mathrm{n}},{ }^{\circ} \mathrm{C}$ \\
\hline Soil with short poor vegetation & 0.75 \\
Soil with $20 \mathrm{~cm}$-high poor vegetation & 0.73 \\
Soil with $20 \mathrm{~cm}$-high thick vegetation & 0.33 \\
Freshly cut lawn & 0.75 \\
Gravel & 0.80 \\
Asphalt & 0.35 \\
Road bricks & 0.50 \\
Concrete & 0.42 \\
\hline
\end{tabular}

\subsection{Surrogate detection}

In our experiments, the landmine surrogates were buried in sand at the depth about 2 $\mathrm{cm}$. The examples of the IR thermograms recorded under natural solar heating are shown in figure 4. The aluminum surrogate looks colder than the background, and the thermal pattern is specific because of the concave shape of the aluminum surrogate. Respectively, the paraffin surrogate looks warmer than the background and its detection is easier due to 
the surrogate round shape. It is worth noting that, due to the low Sun, the natural roughness of the sand surface has produced a significant non-uniform heating pattern.

In order to enhance the defect visibility at 11:30 next day, the site was heated with a $500 \mathrm{~W}$ electrical lamp for $1 \mathrm{hr}$. The source image at the end of heating revealed a weak footprint of the buried mines (figure 5a). The defect visibility was improved a lot after having subtracted the initial image from the image recorded at the end of heating (figure $5 b)$. In order to analyze the influence of sand moisture, the site was moistened in the evening and monitored during the next day. The solar radiation was rather weak $(Q$ changed from 30 to $240 \mathrm{~W} \mathrm{~m}^{-2}$ ) that day. The IR image taken at 15:00 is shown in figure 6 . It is interesting that the background became more uniform than in figure 4 and the mine visibility proved to be a little better. The temperature signals were: $\Delta T_{\mathrm{m}}=+0.9^{\circ} \mathrm{C}$ over the paraffin surrogate and $\Delta T_{m}=-0.3^{\circ} \mathrm{C}$ over the aluminum surrogate.

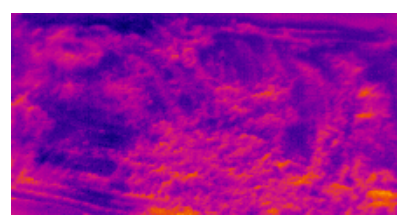

11:00

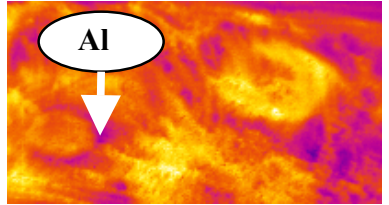

16:00

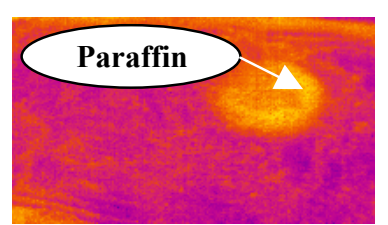

19:30

Fig. 4. Detecting the aluminum and paraffin landmine surrogates buried in sand at the depth of $2 \mathrm{~cm}$ (natural heating)

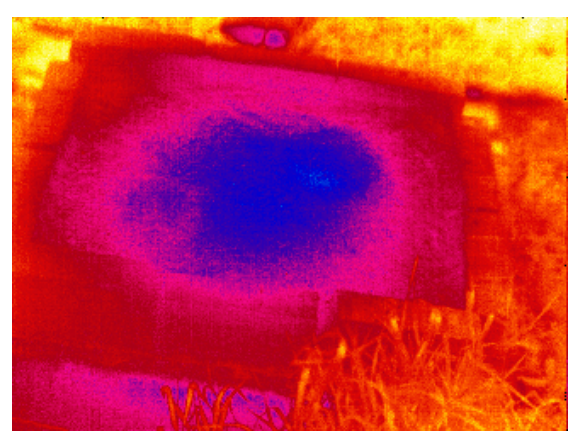

a)

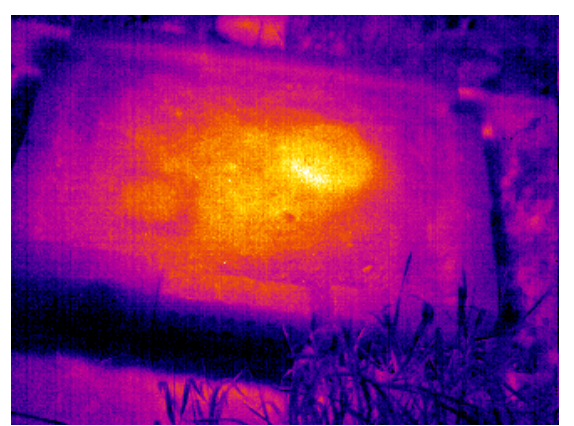

b)

Fig. 5. Detecting the aluminum and paraffin landmine surrogates buried in sand at the depth of $2 \mathrm{~cm}$ (artificial heating for $1 \mathrm{hr}$ with a $500 \mathrm{~W}$ lamp): $a$ - source image, $b$ - same as a) after subtracting the ambient image

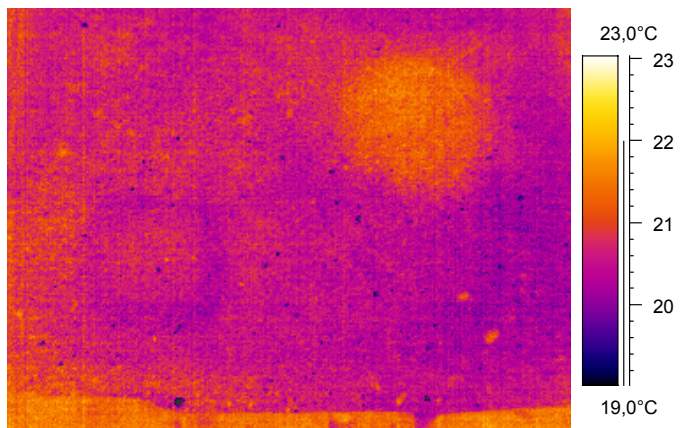

Fig. 6. IR image at $15: 00$ over the moistened sand (natural heating, $Q=30-240 \mathrm{~W} \mathrm{~m}^{-2}$ ) 


\section{Conclusion}

The analysis of the landmine detection results obtained on the aluminum and paraffin surrogates buried in sand has led us to rather pessimistic conclusions about the potentials of using IR thermography for detecting single landmines under varying environmental conditions. First of all, if to assume the detection mechanism to be based exclusively on the difference in the thermal properties between soils and landmines, the appearing temperature signals are quite low in regard to the typical surface noise thus limiting the detection depth up to about $3 \mathrm{~cm}$. Second, a combination of poorly-controlled varying natural parameters, such as wind, moisture, cloudy Sky, etc., might influence adversely on the detection reliability. Third, a low-power thermal stimulation of soil might improve the landmine detection during the periods when natural signals are vanishing; nevertheless, a practical realization of such technique seems to be difficult, especially, from the point of view of inspection productivity. A particular shape of landmines may help in their detection by thermal footprints, however, the best detection results could appear if landmines are distributed in a regular way, as it is used in some mining techniques.

It is worth noting that our results are related to both weak natural heating and high surface noise. The effectiveness of landmine detection might be significantly improved in the case of low geographical latitudes and uniform soil textures, e.g. in a desert.

\section{REFERENCES}

[1] Vavilov, V.P., "Three-dimensional analysis of transient thermal NDT problems by data simulation and processing", Proc.SPIE "Thermosense-XXII", Vol. 4020, 2000, p. $152-160$.

[2] Sendur, I.K. and Baertlein, B.A., "Numerical simulation of thermal signatures of buried mines over a diurnal cycle", Proc. SPIE "Detection and remediation technologies for mines and minelike targets", 2000, p. 144-150. 